Ana Rodrigues Falbo 1 João Guilherme Bezerra Alves 1

\section{Desnutrição grave: alguns aspectos clínicos e epidemiológicos de crianças hospitalizadas no Instituto Materno Infantil de Pernambuco (IMIP), Brasil}

\author{
Severe malnutrition: epidemiological \\ and clinical characteristics of children hospitalized \\ in the Instituto Materno Infantil de Pernambuco \\ (IMIP), Brazil
}

1 Instituto Materno Infantil de Pernambuco. Rua dos Coelhos 300 , Recife, $P E$ 50070-550, Brasil.

\begin{abstract}
Ninety-nine children admitted to the Instituto Materno Infantil de Pernambuco with severe malnutrition from May 1999 to May 2000 were investigated in a cross-sectional study focusing on key epidemiological and clinical variables. The majority of the children (88.9\%) were less than 6 months of age, $42.4 \%$ had a history of low birth weight, and $36.4 \%$ were premature. Some 19.2\% had never been breastfed, and 49.5\% had been breastfed for less than 2 months. Some $15.2 \%$ of the mothers were illiterate. Most of the families (86.1\%) had incomes less than twice the minimum wage (approximately US\$150/month), and 51.5\% had migrated from rural areas. Only 26.3\% of the homes had running water, and $40.4 \%$ lacked sewage disposal facilities. Diarrhea was the reason for hospital admission in 55.6\% of the cases. Hospital mortality was $34.3 \%$ in this group.
\end{abstract}

Key words Nutrition Disorders; Hospitalization; Child Health

Resumo Foram estudadas todas as crianças, hospitalizadas no Instituto Materno Infantil de Pernambuco, com desnutrição grave, no período de maio de 1999 a maio de 2000. O estudo foi de natureza descritiva com desenho transversal. O objetivo do estudo foi conhecer o perfil dessas crianças, em relação a algumas variáveis clínicas e epidemiológicas. A maioria das crianças tinha idade menor que 6 meses (88,9\%), tendo sido observado um percentual de 42,4\% de baixo peso ao nascer e 36,4\% de prematuridade. Dezenove vírgula dois porcento das crianças nunca receberam leite materno, e 49,5\% foram amamentadas por um periodo inferior a 2 meses. Verificou-se que 15,2\% das mães não tinham nenhum grau de escolaridade. A renda familiar foi menor que 2 salários mínimo em 86,1\% das famílias. A maioria das crianças veio do interior do Estado (51,5\%). Em relação ao saneamento básico, 26,3\% das casas não tinham água encanada $e$ em 40,4\% não havia fossa séptica. A diarréia foi o motivo da internação em 55,6\% dos casos. A mortalidade hospitalar no grupo do estudo foi de 34,3\%.

Palavras-chave Desnutrição; Hospitalização; Saúde Infantil 


\section{Introdução}

A desnutrição energético-protéica (DEP) constitui-se num dos principais problemas de saúde coletiva em escala mundial, por sua magnitude, conseqüências biológicas e danos sociais. No nordeste do Brasil, as formas graves de DEP chegam a atingir $24,0 \%$ das crianças menores de 5 anos de idade, hospitalizadas (Alves et al., 1988; Batista Filho et al., 1988). A II Pesquisa Estadual de Saúde e Nutrição (INAN/UFPE/SES$\mathrm{PE}, 1998$ ) encontrou, segundo o indicador peso/idade, um percentual de $4,9 \%$ de desnutrição grave e moderada (abaixo de -2 desviospadrão) em todo o Estado de Pernambuco.

A letalidade hospitalar em menores portadores de formas graves de DEP chega a ser quase dez vezes mais elevada quando comparada com crianças eutróficas (Alves et al., 1988; Guillerman \& Taboadela, 1984; Schofield \& Ashworth, 1997). Estudo realizado no Instituto Materno Infantil de Pernambuco (IMIP), observou que, de 1.045 crianças carentes falecidas no hospital no ano de 1995, 60,1\% tinham algum grau de comprometimento do estado nutricional (Alves et al., 1996). Em 1998, 15,1\% das crianças desnutridas graves que foram hospitalizadas no IMIP evoluíram para o óbito (NEPI, 1998). Esses dados evidenciam, de forma expressiva, o papel do hospital como centro de demanda dos casos de desnutrição grave.

Com o objetivo de se obter um maior conhecimento do problema em nível hospitalar, realizamos esse estudo, que descreve alguns aspectos clínicos e epidemiológicos de crianças com desnutrição grave. Esses dados fornecerão uma base importante para a realização de estudo de intervenção que possa então contribuir de modo mais efetivo para melhorar a abordagem médica da criança com desnutrição grave hospitalizada.

\section{Metodologia}

O estudo foi realizado no IMIP, Recife, Estado de Pernambuco. Como centro de referência do SUS-Pernambuco, esse instituto atende crianças oriundas de todos os municípios do Estado de Pernambuco e também de estados vizinhos.

Em um estudo do tipo seccional, foram pesquisadas todas as crianças hospitalizadas no período de maio de 1999 a maio de 2000 (99 crianças), com idade de um a 60 meses, com desnutrição grave (índice peso/idade < percentil 3). Para a coleta dos dados utilizou-se um formulário construído conforme as variáveis do estudo (idade, sexo, peso ao nascer, idade ges- tacional, aleitamento materno, estado vacinal, local da habitação, água encanada e fossa séptica na habitação, motivo da hospitalização, presença de edema, estado de hidratação e sinais de choque hipovolêmico na admissão, uso de antibiótico e soro para reidratação oral durante a hospitalização), a fim de atender os objetivos da pesquisa. As informações foram coletadas dos prontuários médicos, pois o estudo foi retrospectivo. Para a criação do banco de dados, duas entradas foram realizadas por diferentes digitadores, utilizando-se o programa Epi Info 6.0. A avaliação do estado nutricional foi realizada pelo Epinut do Epi Info 6.0, que utiliza como referência o padrão do National Center for Health Statistics (NCHS, 1977). Verificou-se a distribuição de freqüência das variáveis estudadas, que são apresentadas em forma de tabelas.

\section{Resultados}

Os dados que caracterizam a amostra em relação ao sexo, idade, peso ao nascer, idade gestacional, aleitamento materno e estado vacinal, encontram-se descritos na Tabela 1.

Em relação a algumas variáveis indicadoras da condição da habitação da criança, observou-se que $54,1 \%$ das residências estavam localizadas no interior do Estado; $36,1 \%$ na Zona da Mata. Em 36,1\% das residências não havia água encanada, e constatou-se ausência de fossa séptica em $55,5 \%$ dos casos.

Em 55,6\% das crianças pesquisadas a diarréia foi o motivo da hospitalização, enquanto a pneumonia respondeu por $26,3 \%$ dos casos. Apenas 6,0 \% das crianças do estudo apresentavam edema no momento da admissão hospitalar. Em relação ao estado de hidratação, 32,3\% apresentavam sinais de desidratação do 1 o e $2 \underline{o}$ graus, e 15,2\% do 3 o grau; em 17,2\% dos casos, observaram-se dados sugestivos de choque hipovolêmico. Verificou-se o uso de antibiótico em $81,8 \%$ das crianças hospitalizadas e soro para reidratação oral em $60,0 \%$ delas. Houve evolução para o óbito em $34,3 \%$ das crianças.

Os dados que caracterizam a mostra em relação à idade e escolaridade das mães e à renda familiar, encontram-se descritos na Tabela 2. A taxa de letalidade hospitalar no grupo foi de $34,3 \%$ (34 crianças). As Tabelas 3 e 4 mostram dados que caracterizam este grupo em relação a algumas variáveis clínicas e epidemiológicas, bem como da sua condição sócio-econômica.

As crianças foram sistematicamente classificadas segundo a evolução para alta ou óbito e 


\begin{tabular}{|c|c|c|}
\hline \multicolumn{3}{|c|}{$\begin{array}{l}\text { Distribuição de freqüência das crianças hospitalizadas } \\
\text { com desnutrição no Instituto Materno Infantil } \\
\text { de Pernambuco, segundo algumas variáveis } \\
\text { epidemiológicas. Recife, Pernambuco, Brasil, } \\
\text { 1999-2000. }\end{array}$} \\
\hline Variáveis & $\mathrm{n}$ & $\%$ \\
\hline \multicolumn{3}{|l|}{ Sexo } \\
\hline Masculino & 52 & 52,5 \\
\hline Feminino & 47 & 47,5 \\
\hline \multicolumn{3}{|l|}{ Idade (meses) } \\
\hline 1 a 6 & 88 & 88,9 \\
\hline 6 a 12 & 8 & 8,1 \\
\hline 12 a 24 & 1 & 1,0 \\
\hline$\geq 24$ & 2 & 2,0 \\
\hline \multicolumn{3}{|l|}{ Peso ao nascer $(\mathrm{g})$} \\
\hline$<2.500$ & 42 & 59,2 \\
\hline 2.500 a 3.000 & 17 & 23,9 \\
\hline$\geq 3.000$ & 12 & 16,9 \\
\hline \multicolumn{3}{|l|}{ Idade gestacional } \\
\hline Pré-termo & 36 & 46,1 \\
\hline A termo & 42 & 53,8 \\
\hline \multicolumn{3}{|c|}{ Aleitamento materno (meses) } \\
\hline Nunca mamou & 19 & 22,8 \\
\hline$<2$ & 49 & 59,0 \\
\hline 2 a 4 & 12 & 14,4 \\
\hline 4 a 6 & 3 & 3,6 \\
\hline \multicolumn{3}{|l|}{ Vacinas } \\
\hline Nenhuma & 29 & 56,8 \\
\hline Incompletas p/idade & 14 & 27,4 \\
\hline Completas $\mathrm{p} /$ idade & 8 & 15,6 \\
\hline
\end{tabular}

considerando as variáveis que foram demonstradas anteriormente (Tabelas 3 e 4), e não foram observadas diferenças estatisticamente significantes entre as variáveis, obtendo-se sempre um $\mathrm{p}>0,05$.

\section{Discussão}

Apesar das evidências recentes de redução dos índices de DEP no Brasil, especialmente no Nordeste, ela continua como a endemia carencial mais importante em nosso país, provocando uma elevada demanda nos serviços de saúde e aumento nas taxas de mortalidade hospitalar (Batista Filho \& Costa, 1988). Isso aumenta a relevância do estudo da desnutrição hospitalar,

\begin{tabular}{|c|c|c|}
\hline \multicolumn{3}{|c|}{$\begin{array}{l}\text { Distribuição de freqüência das crianças hospitalizadas } \\
\text { com desnutrição no Instituto Materno Infantil } \\
\text { de Pernambuco, segundo a idade da mãe, } \\
\text { a escolaridade da mãe e a renda. Recife, } \\
\text { Pernambuco, Brasil, 1999-2000. }\end{array}$} \\
\hline Variáveis & $\mathrm{n}$ & $\%$ \\
\hline \multicolumn{3}{|l|}{ Idade da mãe (anos) } \\
\hline$<19$ & 17 & 20,9 \\
\hline 19 a 30 & 49 & 60,5 \\
\hline 30 a 40 & 14 & 17,3 \\
\hline$\geq 40$ & 1 & 1,3 \\
\hline \multicolumn{3}{|l|}{ Escolaridade da mãe } \\
\hline Nenhuma & 15 & 20,3 \\
\hline Ensino fundamental incompleto & 47 & 63,5 \\
\hline Ensino fundamental completo & 8 & 10,8 \\
\hline Ensino médio incompleto & 2 & 2,7 \\
\hline Ensino médio completo & 2 & 2,7 \\
\hline \multicolumn{3}{|l|}{ Renda familiar (SM) } \\
\hline$<1$ & 27 & 41,5 \\
\hline 1 a 2 & 29 & 44,6 \\
\hline 2 a 3 & 2 & 3,0 \\
\hline$>3$ & 7 & 10,9 \\
\hline
\end{tabular}

$\mathrm{SM}=$ Salário Mínimo

pelos seus efeitos desfavoráveis sobre o prognóstico dos pacientes. No presente estudo, a maioria das crianças tinha idade menor que seis meses. Os elevados percentuais de baixo peso ao nascer e prematuridade, ao lado de elevados índices de desmame precoce, contribuem para justificar esse achado.

A ação que mais contribuiria para a prevenção das interações maléficas do binômio DEP/ infecção seria a prática da amamentação exclusiva até o 60 mês de vida. Vários estudos têm comprovado o papel da proteção do leite materno, particularmente em relação às infecções do trato digestivo e respiratório, responsáveis pela grande maioria das hospitalizações em nossa casuística. Nos países em desenvolvimento, crianças não amamentadas no seio têm 3 a 4 vezes mais chances de morrer nos três primeiros meses de vida (Beaudry et al., 1995; Ebrahim, 1995). Infelizmente, a freqüência e a duração do aleitamento materno são baixas em nosso meio, o que contribui para índices bastante elevados de morbimortalidade no primeiro ano de vida (INAN, 1990).

Dentre as crianças estudadas, apenas 15,6\%, tinham esquema vacinal completo. Existe uma forte associação entre ocorrência de doenças 


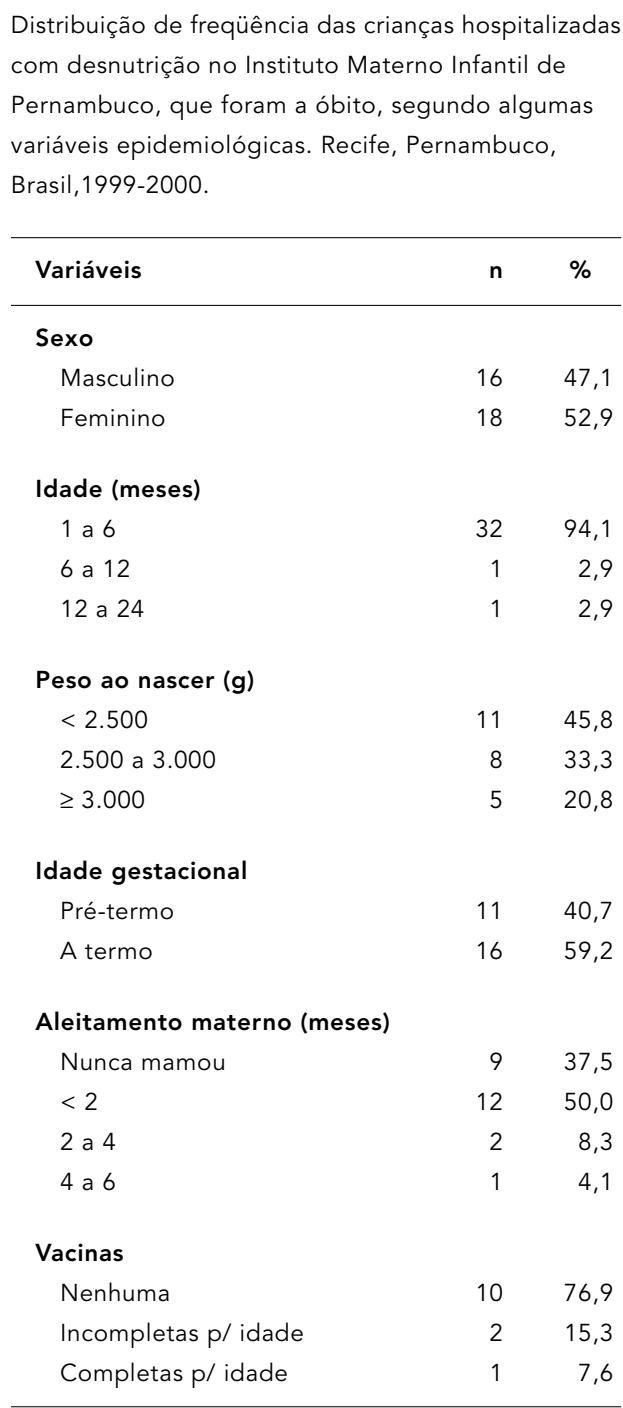

imunopreveníveis e posterior surgimento de doenças infecciosas, sobretudo diarréia e pneumonia, em função da queda de imunidade imposta pelas doenças imunopreveníveis (Deivanayagam et al., 1993; Mahalanabis et al., 1991).

No presente estudo, foi elevado o número de mães adolescentes e com baixa escolaridade, reconhecidamente fatores de risco para agravos à saúde (Victora et al., 1993). A baixa renda das famílias estudadas aponta para uma situação de miséria. Evidentemente, essa situação gera condições nutricionais insatisfatórias. Estima-se, hoje, que $50,95 \%$ da população do Estado de Pernambuco tenham uma renda mensal inferior a $\mathrm{R} \$ 80,00$ per capita (Neri et al., 2000).

Dos domicílios das crianças do estudo, 36,1\% não tinham acesso à água encanada e 55,5\%

\begin{tabular}{|c|c|c|}
\hline \multicolumn{3}{|c|}{$\begin{array}{l}\text { Distribuição de freqüência das crianças hospitalizadas } \\
\text { com desnutrição no Instituto Materno Infantil de } \\
\text { Pernambuco, que foram a óbito, segundo a idade } \\
\text { da mãe, a escolaridade da mãe e a renda. } \\
\text { Recife, Pernambuco, Brasil, 1999-2000. }\end{array}$} \\
\hline Variáveis & n & $\%$ \\
\hline \multicolumn{3}{|l|}{ Idade da mãe (anos) } \\
\hline$<19$ & 9 & 34,6 \\
\hline 19 a 30 & 9 & 34,6 \\
\hline 30 a 40 & 8 & 30,7 \\
\hline \multicolumn{3}{|l|}{ Escolaridade da mãe } \\
\hline Nenhuma & 4 & 16,6 \\
\hline Ensino fundamental incompleto & 17 & 70,8 \\
\hline Ensino fundamental completo & 3 & 12,5 \\
\hline \multicolumn{3}{|l|}{ Renda familiar (SM) } \\
\hline$<1$ & 11 & 50,0 \\
\hline 1 a 2 & 9 & 40,9 \\
\hline$>3$ & 2 & 9,0 \\
\hline
\end{tabular}

$\mathrm{SM}=$ Salário Mínimo

não possuíam fossa séptica. Em termos de saúde ambiental, a falta de acesso a algum tipo de abastecimento de água limpa e de destino adequado dos dejetos, e a manipulação dos alimentos sem cuidados de higiene têm implicações significativas na disseminação de doenças infecciosas.

Apesar de a desnutrição edematosa ser um achado freqüente em muitos países em desenvolvimento, e de essas crianças apresentarem uma evolução com pior prognóstico (Golden, 1996; Manary \& Brewster, 1997; Waterlow, 1992), apenas $6,1 \%$ das crianças estudadas apresentavam edema na admissão hospitalar. A criança com desnutrição grave está propensa a desenvolver sérios problemas que a coloca sob o risco de morrer, dos quais, dentre outros, destacam-se a infecção e os distúrbios hidroeletrolíticos (Schofield \& Ashworth, 1997; WHO, 2000). Das crianças estudadas, $15,2 \%$ chegaram ao serviço apresentando desidratação grave, e outras $17,2 \%$ com sinais de choque hipovolêmico. O grande percentual do uso de antibióticos $(81,8 \%)$ condiz com o fato de que a infecção foi provavelmente um evento comum nessas crianças. Diarréia e pneumonia foram as infecções mais triviais, achados bem descritos em crianças com desnutrição grave (Alves et al., 1988).

Observou-se uma elevada taxa de mortalidade no grupo estudado, de $34,3 \%$, semelhante aos dados citados na literatura, que relatam 
percentuais de mortalidade hospitalar nessas crianças, variando de 20 a 30\% (Schofield \& Ashworth, 1997). O fato de não terem sido observadas diferenças com significância estatística entre algumas variáveis em relação às crianças que receberam alta e as que evoluíram para o óbito, talvez seja explicado pelo manejo dispensado ao grupo como um todo, que não levava em conta as limitações fisiopatológicas inerentes à desnutrição. Este fato aponta para a necessidade de melhorar a abordagem médica dessas crianças.

\section{Referências}

ALVES, J. G. B.; BRITTO, L. M. A.; MELO, M. A. S. \& OLIVEIRA, V. A., 1988. Morbimortalidade no desnutrido grave. Jornal de Pediatria, 64:60-61.

ALVES, J. G. B.; SILVEIRA, V. A. L. \& ANDRADE, T. P., 1996. Obituário em crianças no IMIP - 1995. Revista do IMIP, 10:115-119.

BATISTA FILHO, M. \& COSTA, M. J., 1988. Desnutrição energético-protéica: Experiência do Hospital Universitário de João Pessoa, PB. I - Prevalência da Desnutrição. Revista do IMIP, 2:90-93.

BEAUDRY, M.; DUFOUR, R. \& MARCOUX, S., 1995. Relation between infant feeding and infections during the first six months of life. Journal of Pediatrics, 162:191-197.

DEIVANAYAGAM, N.; MALA, N. \& ASHOK, T. P., 1993. Risk factors for persistent diarrhea among children under 2 years of age, case control study. Indian Pediatrics, 30:177-185.

EBRAHIM, G. J., 1995. Breast milk immunology. Journal of Tropical Pediatrics, 53:2-4.

GOLDEN, M. H. N., 1996. Severe malnutrition. In: $O x-$ ford Textbook of Medicine (D. J. Weatherall, J. G. G. Ledingham \& D. A. Warrell, ed.), pp. 1278-1294, 3rd Ed. Oxford: University Oxford Press.

GUILHERME, S. N. \& TABOADELA, M., 1984. Evaluación del estado nutricional de pacientes internados en un hospital pediátrico. Archivos Argentinos de Pediatría, 82:393-402.

INAN (Instituto Nacional de Alimentação e Nutrição) / IMIP (Instituto Materno Infantil de Pernambuco)/ UFPE (Universidade Federal de Pernambuco)/ SES-PE (Secretaria Estadual de Saúde do Estado de Pernambuco), 1998. Saúde, Nutrição, Alimentação e Condições Sócio-econômicas no Estado de Pernambuco. II Pesquisa Estadual de Saúde e Nutrição. Recife: INAN/Ministério da Saúde/Departamento de Nutrição, UFPE/SES-PE.
Portanto, a maior parte das crianças tinha idade inferior a 6 meses, precárias condições de habitação e foi desmamada precocemente. Mais de um terço delas teve nascimento prematuro, baixo peso ao nascer e evolução para o óbito. O conhecimento dessas características clínicas e epidemiológicas nos forneceu subsídios para desenvolver um estudo de intervenção, visando testar, em nosso meio, a aplicação do protocolo da OMS para manejo da desnutrição grave, cujos principais objetivos são a redução do risco de óbito e a diminuição da permanência hospitalar.
MAHALANABIS, D.; ALAN, A. N. \& RAHMAN, N., 1991. Prognostic indicators and risk factors for increased duration of acute diarrhea and for persistent diarrhea in children. International Journal of Epidemiology, 20:1064-1072.

MANARY, M. J. \& BREWSTER, D. R., 1997. Potassium supplementation in Kwashiorkor. Journal of Pediatric Gastroenterology and Nutrition, 24:194-201.

NCHS (National Center of Health Statistics), 1977. Growth curves for children. Birth-18 years. Vital Health Statistics, 11:1-74.

NERI, M.; GONZAGA, G. \& CAMARGO, J. M., 2000. Efeitos Informais do Salário Mínimo e Pobreza. Rio de Janeiro: Centro de Políticas Sociais, Fundação Getúlio Vargas.

NEPI (Núcleo de Epidemiologia), 1998. Boletim Epidemiológico Semestral. Recife: Hospital Geral de Pediatria, Instituto Materno Infantil de Pernambuco.

SCHOFIELD, C. \& ASHWORTH, A., 1997. Por qué siguen tan altas las tasas de mortalidad por malnutrición grave? Revista Panamericana de Salud Pública, 1:295-299.

VICTORA, C. G.; HUTTLY, S. R. \& FUCHS, S. C., 1993. International differences in clinical patterns of diarrhoeal deaths: A comparison of children from Brazil, Senegal, Bangladesh and India. Journal of Diarrhoeal Diseases Research, 11:25-29.

WATERLOW, J. C., 1992. Protein-energy Malnutrition. London: Arnold.

WHO (World Health Organization), 2000. Management of the Child with a Serious Infection or Severe Malnutrition. Geneva: WHO.

Recebido em 14 de agosto de 2001

Versão final reapresentada em 26 de março de 2002

Aprovado em 10 de maio de 2002 